\title{
Exerting self-control for persistence
}

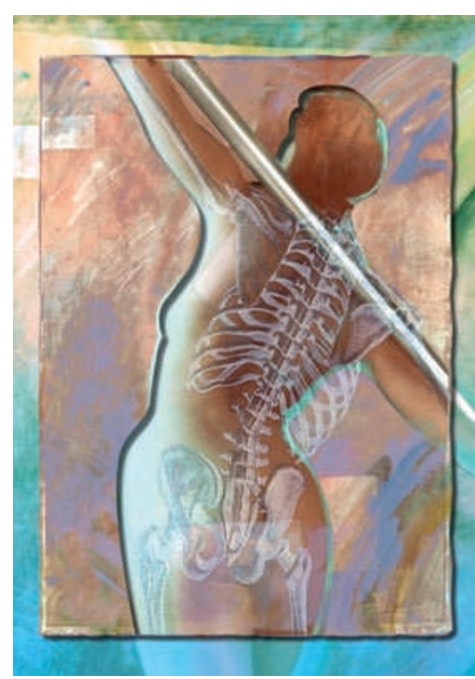

Synapses made by a neuron onto itself — known as autapses - have been described in various brain regions, including the neocortex, hippocampus, cerebellum, substantia nigra and striatum, but their purpose has remained uncertain. Reporting in Current Biology, Susswein and colleagues show that autaptic transmission in Aplysia B31/B32 neurons of the buccal ganglia is involved in the long-lasting response to brief stimulation.
The activity of B31/B32 neurons is required to initiate and maintain feeding behaviour in sea slugs, but the mechanism whereby their activity outlasts brief depolarization stimuli is not fully understood. The B63 neuron has been shown to excite B31/B32 neurons in situ, through electrical coupling and cholinergic neurotransmission. Here, the authors showed that fast B63-elicited excitatory postsynaptic potentials (EPSPs) in B31/B32 neurons were blocked by the nicotinic antagonist hexamethonium, and that slow EPSPs, which lead to a sustained plateau depolarization, were blocked by the muscarinic antagonist pirenzepine. However, the lasting depolarization of B31/B32 neurons ocurred even in response to pulses that were too weak to stimulate B63 firing, suggesting that the persistent activity of the B31/B32 neurons is not completely dependent on presynaptic input but might involve voltagedependent muscarinic autaptic transmission. Indeed, further anatomical characterization revealed that B31/B32 neurons exhibit highly branched neurites with varicosities close to the soma, which are likely to be autaptic presynaptic release sites.
The persistent activity of B31/B32 neurons following brief stimulation was replicated in vitro, and here, as in situ, pirenzepine blocked persistent activity. To demonstrate that these B31/B32 autapses are not an artefact of culturing the cells in isolation, the authors showed that other buccal ganglia neurons do not develop plateau potentials in vitro, and that even when B31/B32 neurons were not in isolation but co-cultured with other neurons, autapse-based plateau potentials still developed. In fact, depolarizing one B31/B32 neuron often caused persistent activity only in the stimulated cell, suggesting that these cells actually prefer to form synapses with themselves.

This study helps to refute the idea that autapses are artefacts of growing neurons in isolation and highlights an important role for this type of synapse in the maintenance of persistent activity.

Monica Hoyos Flight

ORIGINAL RESEARCH PAPER Saada, R., Miller, N., Hurwitz, I. \& Susswein, A. J. Autaptic excitation elicits persistent activity and a plateau potential in a neuron of known behavioral function. Curr. Biol. 19, 479-484 (2009) 